\title{
CARDIOPROTECTIVE POTENTIAL OF PLANTS AND PLANT-DERIVED PRINCIPLES - A REVIEW
}

\author{
NIKITHA G, RAJENDRA SANDUR V*
}

Department of Pharmacology, Krupanidhi College of Pharmacy, Bengaluru, Karnataka, India. Email: drrajendra1972@gmail.com

\author{
Received: 26 November 2018, Revised and Accepted: 15 January 2019
}

\begin{abstract}
Cardiovascular diseases (CVDs) are a class of diseases involving heart or blood vessels. Coronary artery diseases include angina, myocardial infarction (MI), stroke, heart failure, hypertensive heart disease, cardiomyopathy, and arrhythmias. CVDs are the leading cause of death globally. Risk factors include high blood pressure, smoking, obesity, poor diet, blood cholesterol, and lack of exercise. It is approximated that $90 \%$ of CVDs is preventable. High blood pressure results in 13\% of CVD deaths, whereas tobacco outcomes in 9\%, diabetes and lack of exercise in around $6 \%$, and obesity in 5\%. Due to certain medications such as anticancer drugs like doxorubicin, adverse effects result in MI. Since ancient times, medicinal plants have been widely used in the treatment of diseases. This information may serve as a primer in identifying novel prophylactic as well as therapeutic studies of plant-derived principles. The parts of the plants such as seeds, leaves, flowers, roots, and bark contain these phytoconstituents which are obtained through different extraction processes. Phytoconstituents are broadly classified into alkaloids, saponins, polyphenols, essential oils, carotenoids, glycosides, omega fatty acids, and flavonoids. Each class is responsible for its own pharmacological effects. The underlying mechanism in which they exert the action is different. This review presents an overview of the MI and therapeutic strategies of plant-derived principles that are available to mitigate the effect of MI.
\end{abstract}

Keywords: Cardiovascular diseases, Myocardial infarction, Medicinal plants, Phytoconstituents, Alkaloids, Saponins, Sulfur-containing compounds, Carotenoids, Flavanoids, Essential oils, Polyphenols.

(C) 2019 The Authors. Published by Innovare Academic Sciences Pvt Ltd. This is an open access article under the CC BY license (http://creativecommons. org/licenses/by/4. 0/) DOI: http://dx.doi.org/10.22159/ajpcr.2019.v12i3.31011

\section{INTRODUCTION}

Medicinal plants, also called medicinal herbs, have been identified and used in ancient medicine practices since prehistoric times. Plants synthesize hundreds of chemical compounds for functions including defense against insects, fungi, diseases, and herbivorous mammals [1]. There are four major biochemical classes of phytoconstituents found in plants which include alkaloids, glycosides, polyphenols, and terpenes. Medicinal plants are widely used in underdeveloped societies, mainly because they are readily available and cheaper than the modern medicines. Cardiovascular disease (CVD), predominantly myocardial infarction (MI), is one of the leading causes of death worldwide [2]. Acute MI is the most important and serious consequence of coronary artery disease. It occurs when myocardial ischemia, decreased blood supply to heart, exceeds a critical threshold and overwhelms myocardial cellular repair mechanisms designed to maintain normal operating function and homeostasis [3]. MI is invariably followed by numerous pathophysiological and biochemical alterations including hyperlipidemia, thrombosis, lipid peroxidation (LPO), and free radicle damage, leading to qualitative and quantitative changes of myocardium [4]. Although clinical care is improvised, public awareness is raised, and health innovations are widely used, MI still remains the leading cause of death worldwide [5].

\section{Secondary metabolites and their cardioprotective role}

Secondary metabolites are organic compounds synthesized by plants which are not directly involved in the normal growth, development, or reproduction of the organism. One of the most important defining qualities of secondary metabolites is their specificity [6]. There are a number of plants containing various classes of phytoconstituents responsible for cardioprotective activity as summarized in Table 1.

\section{Role of secondary metabolites in cardioprotection}

Alkaloids

Alkaloids are a class of naturally occurring phytoconstituents that mostly contain basic nitrogen atoms. In addition to carbon, hydrogen, and nitrogen, alkaloids may also contain oxygen, sulfur and, more rarely, other elements such as chlorine, bromine, and phosphorus [7]. Alkaloids are synthesized by a large variety of organisms including bacteria, fungi, plants, and animals [8]. They can be obtained from the purification of the crude extracts of these organisms by acid-base extraction. Alkaloids have a wide range of pharmacological activities including antimalarial [9], anticancer [10], vasodilatory [11], antiarrhythmic [12], analgesic [13], antibacterial [14], and antidiabetic [15] activities.

\section{Tinosporin}

Tinospora cordifolia is an herbaceous vine of the family Menispermaceae which is indigenous to the tropical areas of India, Sri Lanka, and Myanmar. Also called Guduchi in Ayurvedic medicine, a variety of active components such as alkaloids, steroids, diterpenoid lactones, aliphatics, and glycosides have been isolated from the different parts of the plant body, including root, stem, and the whole plant [16]. The alkaloids which are predominantly found in T. cordifolia include tinosporin (Table 2), berberine, and palmatine which are present in the stems. Dose-dependent reductions in infarct size and in serum and heart lipid peroxide levels were perceived significantly with prior treatment of the extract in ischemia-reperfusion (I/R)-induced MI in rats [17]. Physical parameters include gross examination of the heart and heart weight/ body weight ratio and in biochemical estimation, the various cardiac enzymes such as aspartate transaminase (AST), lactate dehydrogenase (LDH), alanine transaminase (ALT), creatinine kinase (CK), and gold marker troponin I were determined. It was observed that there was a significant increase in the level of marker enzymes (AST, ALT, LDH, and TROPONIN) in the serum of isoprenaline-treated rats. When compared with the control group, there was an increase in the level of AST, ALT, $\mathrm{LDH}$, and CK-MB in isoprenaline-induced rats. This finding could be a consequence of decrease in the number of viable myocytes due to augmented cell death in the heart as these animals showed an elevated level of AST, ALT, LDH, and CK-MB. This indicates that T. cordifolia could protect against myocardial damage. The mechanism in which it exerts the effect is by its potentiality to strengthen the myocardial damage by its membrane-stabilizing action [18]. Alkaloids found in other plants 
Table 1: List of plants and their phytoconstituents responsible for cardioprotection

\begin{tabular}{lll}
\hline Phytoconstituents & Plant name & Family \\
\hline Mangiferin [19] & Mangifera indica & Anacardiaceae \\
Allicin [20] & Allium sativum & Amaryllidaceae \\
Eugenol [21] & Ocimum basilicum & Lamiaceae \\
Sesamol [22] & Sesamumindicum & Pedaliaceae \\
Arjunolic acid [23] & Terminalia arjuna & Combretaceae \\
Gingerol [21] & Zingiber officinale & Zingiberaceae \\
Hesperidin [24] & Citrus aurantium & Rutaceae \\
Curcumin [25] & Curcuma longa & Zingiberaceae \\
Resveratrol [26] & Vaccinium corymbosum & Ericaceae \\
Proanthocyanidins [27] & Crataegus oxycantha & Rosaceae \\
$\alpha$-linolenic acid [28] & Linumusitatissimum & Linaceae \\
Oleuropin [29] & Oleaeuropeae & Oleaceae \\
Ginsenosides [30] & Panax ginseng & Araliaceae \\
Avicularin [31] & Polygonumaviculare & Polygonaceae \\
Flavanoids [32] & Myrciariacauliflora & Myrtacea \\
Thymoquinone [33] & Nigella sativa & Rananculaceae \\
Lycopene [34] & Solanumlycopersicum & Solanaceae \\
Silymarin [35] & Silybummarianum & Asteraceae \\
\hline
\end{tabular}

such as Coriandrum sativum, Newbouldia laevis, Nigella sativa, and Tamrindus indica are also cardioprotective in nature [28].

\section{Vincristine (vinca alkaloids)}

Studies conducted revealed that pretreatment with vincristine (Table 2) significantly reduced the activities of marker enzymes such as CK$\mathrm{MB}$, serum glutamate pyruvate transaminase and LDH, and the levels of Troponin-T in the serum. Elimination of Isoproterenol-induced histopathological changes and reduction in myocardial necrosis were seen by transmission electron microscopy findings due to the pretreatment of vincristine. Thus, vincristine acts as a potent alkaloidal agent in attenuating isoproterenol-induced myocardial necrosis, and the probable mechanism is due to its antioxidant and free radical scavenging activities [36]. Studies explained that simultaneous vincristine treatment provided recovery of the cultured adult mouse myocytes exposed to doxorubicin. Despite the presence of doxorubicin, vincristine retains the ability to activate MAPK pathways. Acceleration of these pathways has been found to provide cardioprotection, especially after preconditioning [37]. Thus, vincristine acts as a potential agent in attenuating isoprenaline-induced cardiotoxicity and doxorubicininduced cardiomyopathy.

\section{Neferine}

Nelumbo lucifera Gaertn, known as lotus belonging to the family Nelumbonaceae, is an aquatic plant from which neferine (Table 2) is obtained. Neferine is a bisbenzylisoquinoline alkaloid. The other active constituents of $N$. lucifera include roemerine, nelumbine, dauricine, liensinine, isoliensinine, lotusine, and nuciferine. Neferine constitutes the major isoquinoline alkaloid along with liensinine and isoliensinine. Previous studies reported the anticancer and antiarrhythmic effects of N. lucifera [38]. Studies reported the activity of neferine against isoproterenol-induced myocardial damage, and it was found that neferine attenuates myocardial damage by acting as a strong antioxidant agent and can be used as a potent agent in oxidative stress [39]. It also toned down the increase of vascular smooth muscle cells and remarkably prevented belomycin-induced pulmonary fibrosis.

\section{Berberine}

Berberine (Table 2), obtained from Rhizomacoptidis belonging to the class of isoquinoline alkaloids, possesses a wide range of pharmacological activities such as antimicrobial, antidiarrheal, antidiabetic, antihyperlipidemic, anti-inflammatory, and antiproliferative effects [40]. Studies conducted revealed that berberine fortified the heart against I/R injury by improvising cardiac function, lowering myocardial apoptosis, and diminishing myocardial dysfunction. Berberine showed its action by preventing apoptosis and improving mitochondrial dysfunction following I/R [41]. Studies conducted by Lv et al. discovered that berberine acts as an potent compound in inhibiting doxorubicintriggered cardiomyocyte apoptosis [42].

\section{Saponins}

Saponins are a class of phytoconstituents found in particular abundance in various plant species. They are amphipathic glycosides, structurally having one or more hydrophilic glycoside moieties combined with a lipophilic triterpene derivative. They produce foam when shaken in aqueous solution [43]. The plants such as Terminalia arjuna, Glycyrrhiza glabra, Ilex cornuta, Crataegus oxyacantha, and Astragalus membranaceus are saponin-enriched plants studied for the cardioprotective activity. Diosgenin and its derivatives which are phytosterols act as a cardioprotective agent by lowering the serum cholesterol by inhibiting the cholesterol absorption in the intestinal tract [44]. Saponins' cardioprotective potential was observed by different pharmacological effects such as antioxidant, anti-hypoxic, anoxia/reoxygenation, $\mathrm{Ca}^{2+}$ ion regulation or calcium antagonist, cardiocyte apoptosis, vasodilatory effect, and angiogenesis, as summarized in Fig. 1 [45].

Diosgenin (Table 2) acts as a good antihyperlipidemic agent. It promotes bone development by significantly elevating the production and secretion of Type I collagen [46]. Earlier studies reported the use of diosgenin in attenuating isoproterenol-induced myocardial damage. Due to its antioxidant property [17], this reversed the membrane-bound enzyme activity and hence helped in maintaining the electrolyte concentration which was the possible mechanism in which it showed its cardioprotective activity [47]. Anti-carcinogenic and antiviral activities [44] of diosgenin were reported earlier, and this was due to its ability in significantly lowering the viral ribonucleic acid and viral proteins [48].

\section{Glycyrrhizic acid}

Glycyrrhizic acid (Table 2) is the major therapeutic constituent obtained from the root extract of the plant G. glabra, also known as liquorice, a shrub from Leguminosae family. It is a triterpene saponin glycoside. Studies revealed that glycyrrhizic acid shows different pharmacological action such as anti-ulcer, expectorant, antiviral, antiinflammatory, antidiabetic, neuroprotective, and immune-enhancing properties. In vivo studies revealed that glycyrrhizic acid possesses antioxidant properties and gives protection against isoproterenolinduced myocardial damage. The possible mechanism in which it exerts action is due to its antioxidant nature and also by a significant reduction in the levels of lipid hydroperoxides and isoprostanes and significant increase in the levels of superoxide dismutase (SOD) and glutathione (GSH) level [49].

\section{Sulfur-containing compounds}

Allicin (Table 2), an organosulfur compound, is obtained from Allium sativum which is commonly known as garlic belonging to the family Amaryllidaceae. When fresh garlic is chopped or crushed, the enzyme allinase converts alliin into allicin, which is responsible for the characteristic aroma of fresh garlic. The allicin formed is unstable and quickly converts into a series of other sulfur-containing compounds such as diallyl disulfide. Allicin is part of a defense mechanism against attacks by pests on the garlic plant [8]. A. sativum, also known as garlic, is said to have originated 6000 years ago and was considered as a good therapeutic potential for treating cardiovascular problems. Other therapeutic properties of this plant include antimicrobial and anticancer and treating other health disorders. In olden days, garlic was used as a good flavoring agent. From the previous studies, two studies reported the efficacy of garlic in the prevention of cardiac hypertrophy in in vivo models of hypertension and diabetes. However, the mechanism in which it showed their actions has not been explored [50]. Preclinical and clinical studies have shown that daily intake of garlic as a food supplement reduces cholesterol and blood pressure along with the inhibition of platelet aggregation. It has also been reported that the vascular function is improved; however, more researches need to 


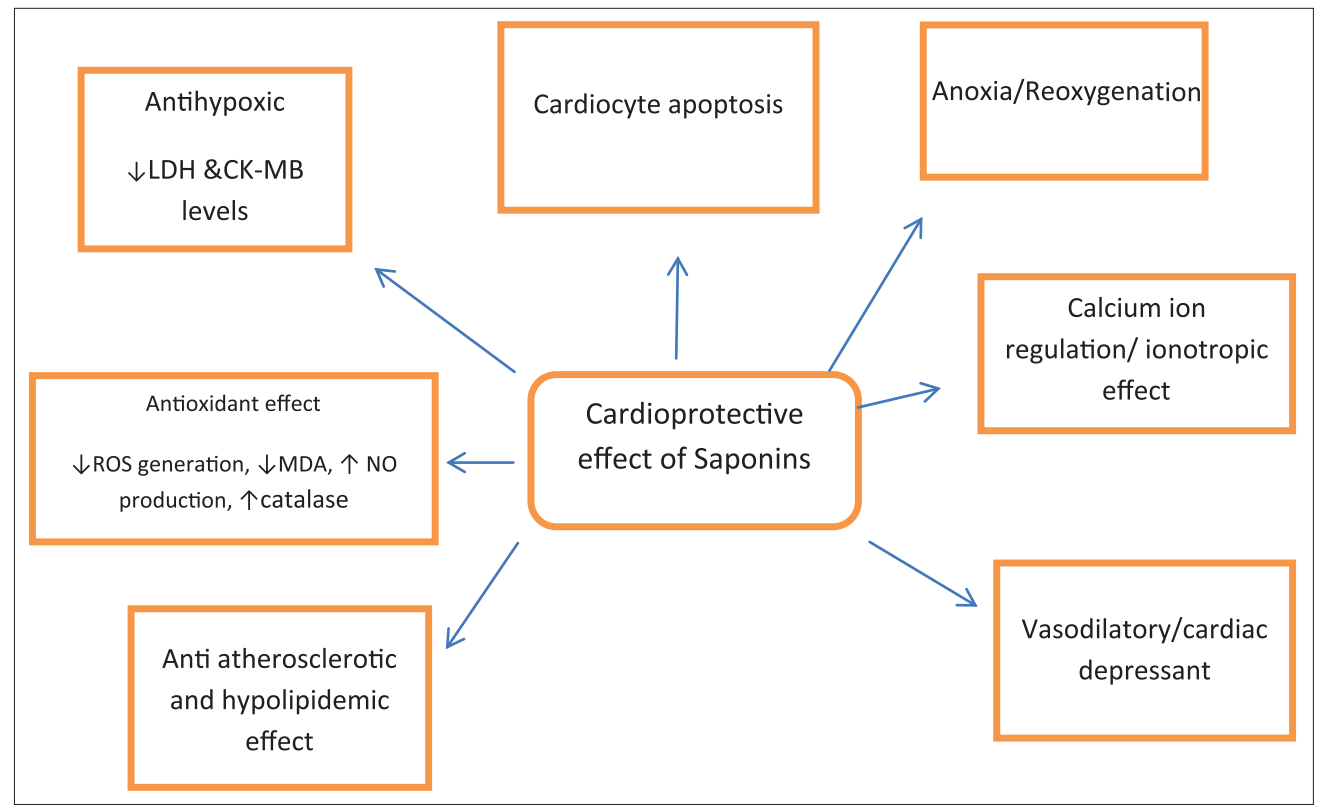

Fig. 1: Mechanism in which saponins show cardioprotection

be done in this area to elaborate on its cardiovascular property. The influence of garlic on heart health is due to the presence of its active metabolite allicin and its breakdown into organic polysulfides. It is hypothesized that hydrogen sulfide may have an important role in garlic-induced cardiac protection. The foremost mechanism by which garlic supplements $\mathrm{H}_{2} \mathrm{~S}$ bioavailability is via alteration of garlic-derived polysulfides. Organic sulfides present in high concentrations in garlic interact readily with thiol groups or thiol-containing compounds (i.e., GSH) present in biological systems to generate free $\mathrm{H}_{2} \mathrm{~S}$. Advanced research suggests that the cardioprotective role of garlic depends on the consumption of the different preparations such as raw garlic, aged garlic, garlic powder, and garlic oil [51].

\section{Essential oils}

Essential oils are also called volatile oils, ethereal oil, or the oil obtained from the plants. They are usually concentrated liquid which are hydrophobic in nature containing volatile aromatic compounds of the plants. They are often referred as essential oils because it contains the "essence" of the plant fragrance, the characteristic fragrance of the particular plant. The methods of extracting the essential oils from plants include mainly steam distillation followed by expression, solvent extraction, absolute oil extraction, resin tapping, wax embedding, and cold pressing. Essential oils are often used in aromatherapy or as an alternative medicine in the treatment of diseases, and the activities shown are due to the presence of compounds responsible for aroma [52]. The commonly available plants from which volatile oils are obtained include coriander, clove, ginger, mentha, spearmint, peppermint, cedar wood, eucalyptus, lemon, cinnamon, and tulsi.

\section{Linalool}

Lavandulaangustifolia, commonly known by the name English Lavender or True Lavender, is a flowering plant belonging to the family Lamiacea. Lavender oil obtained from the plants mainly consists of linalyl acetate, linalool (Table 2), 1,8-cineol, lavandulol, lavandulyl acetate, camphor, cis- $\beta$-ocimene, trans- $\beta$-ocimene, 1 -terpinen- 4 -ol, $\alpha$-terpineol, limonene, tannins, coumarins, flavonoids (luteolin), phytosterols, and triterpens $[53,54]$. L. angustifolia has been used for its wide therapeutic properties such as carminative, diuretic, antiepileptic, antirheumatic and pain reliever, especially in nervous headache and migraine [55]. Lavander essential oil is believed to show potential therapeutic activity against stress, anxiety, exhaustion, irritability, insomnia, depression, colds, digestion, flatulence, upset stomach, liver and gallbladder problems, nervousness, and loss of appetite [56]. The mechanism of action through which the essential oil of L. angustifolia exerts its effect is by anti-inflammatory, free radical scavenging, and antioxidant activities [57]. Thus, from the in vivo studies conducted, it was concluded that the essential oil of L. angustifolia showed cardioprotective activity by normalizing ECG, improving the hemodynamic impairment, reducing LPO, inhibiting pro-inflammatory responses, and boosting the antioxidant defense systems. Essential oil of lavandula maintained the structure and architecture of cardiac cells by decreasing cardiac tissue damage and strengthening myocardial membrane as evident from the histopathological results of myocardial tissue [58].

\section{Coriandrol}

Other plants containing essential oil in which cardioprotection has already been reported include Coriandrum sativa, which belongs to the family Apiaceae commonly known by the name cilantro having the major active constituent coriandrol (Table 2), mainly shows its mechanism of action by the significant reduction in the levels of total cholesterol (TC) and triglycerides (TG) [59]. In an another study, there was a notable decrease in the levels of TC, TG, TAG, and low-density lipoprotein (LDL) cholesterol in plasma and a consequential increase in the level of high-density lipoprotein cholesterol plasma which is discovered in the cholesterol-rich (1\%) basal diet-fed rats treated with coriander seed oil [60]. Antiplatelet activity of the leaf extract of $C$. sativum leaves and curry leaves was monitored at varying concentrations on human platelets, which showed the inhibition of platelet aggregation [61].

Gingerol

Zingiberofficinale, also known as ginger belonging to the family Zingeberaceae and containing the essential oil gingerol (Table 2), is responsible for relaxing the blood vessels, relieving pain, and stimulating blood flow. This acts as a potent anti-inflammatory agent, and hence it is useful in the treatment of cancer, heart diseases, and neurodegenerative disorders such as Alzheimer's disease [61]. Other plants containing volatile oils include cinnamon, black pepper [55], and saffron [62] in which cardioprotective activity has been reported.

\section{Omega fatty acids}

Omega fatty acids are a class of polyunsaturated fatty acids, with the exception being omega 9 fatty acid which is a monounsaturated fatty acid, i.e., having only one double bond. The different omega fatty acids include omega 3 fatty acids, omega 6 fatty acids, and omega 9 fatty acids. The source of omega 3 fatty acids includes salmon, mackerel, sardines, and chia seeds walnuts. Omega 6 fatty acids are obtained from soybean 

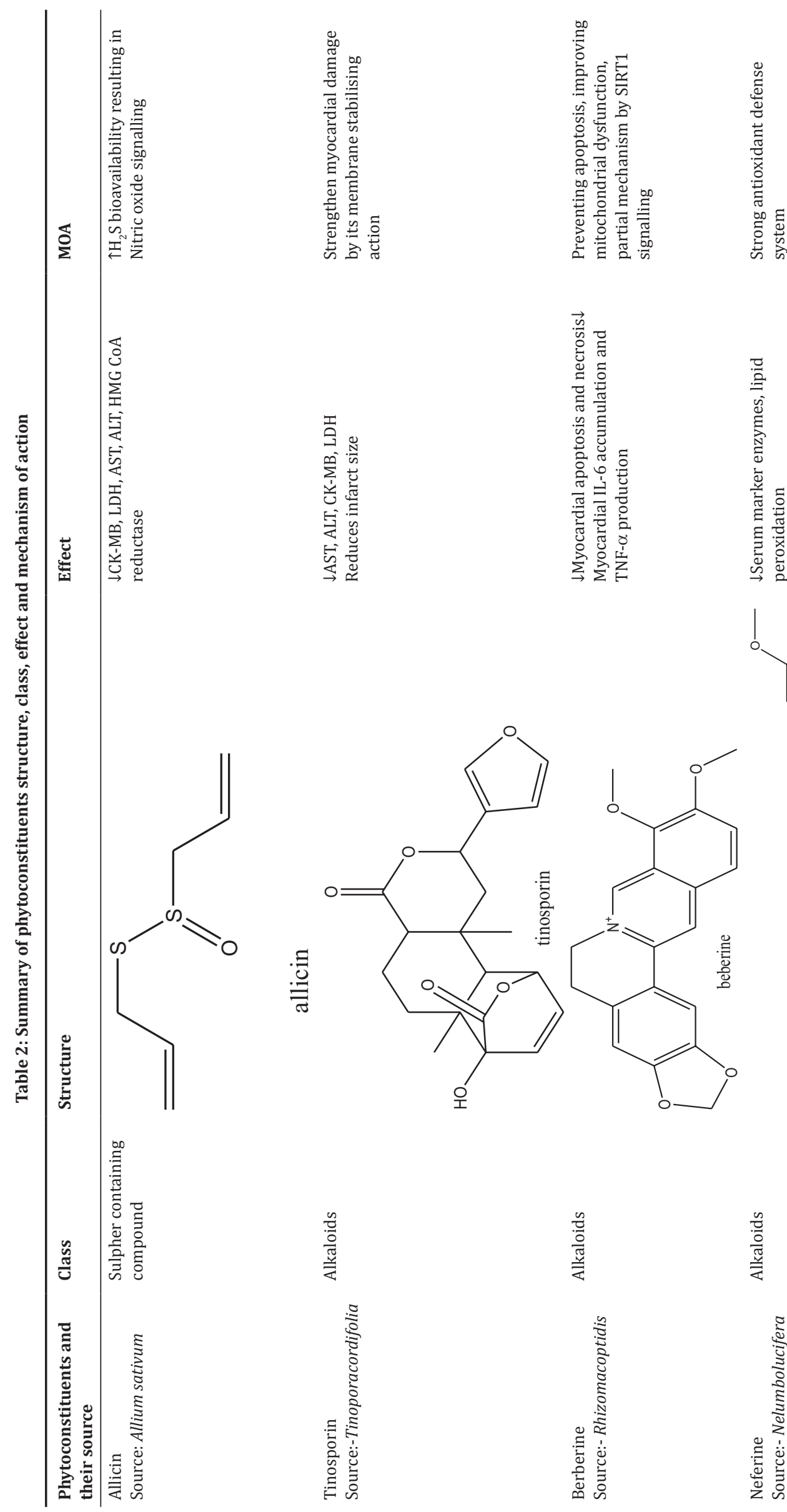

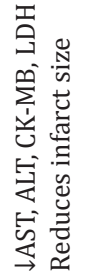
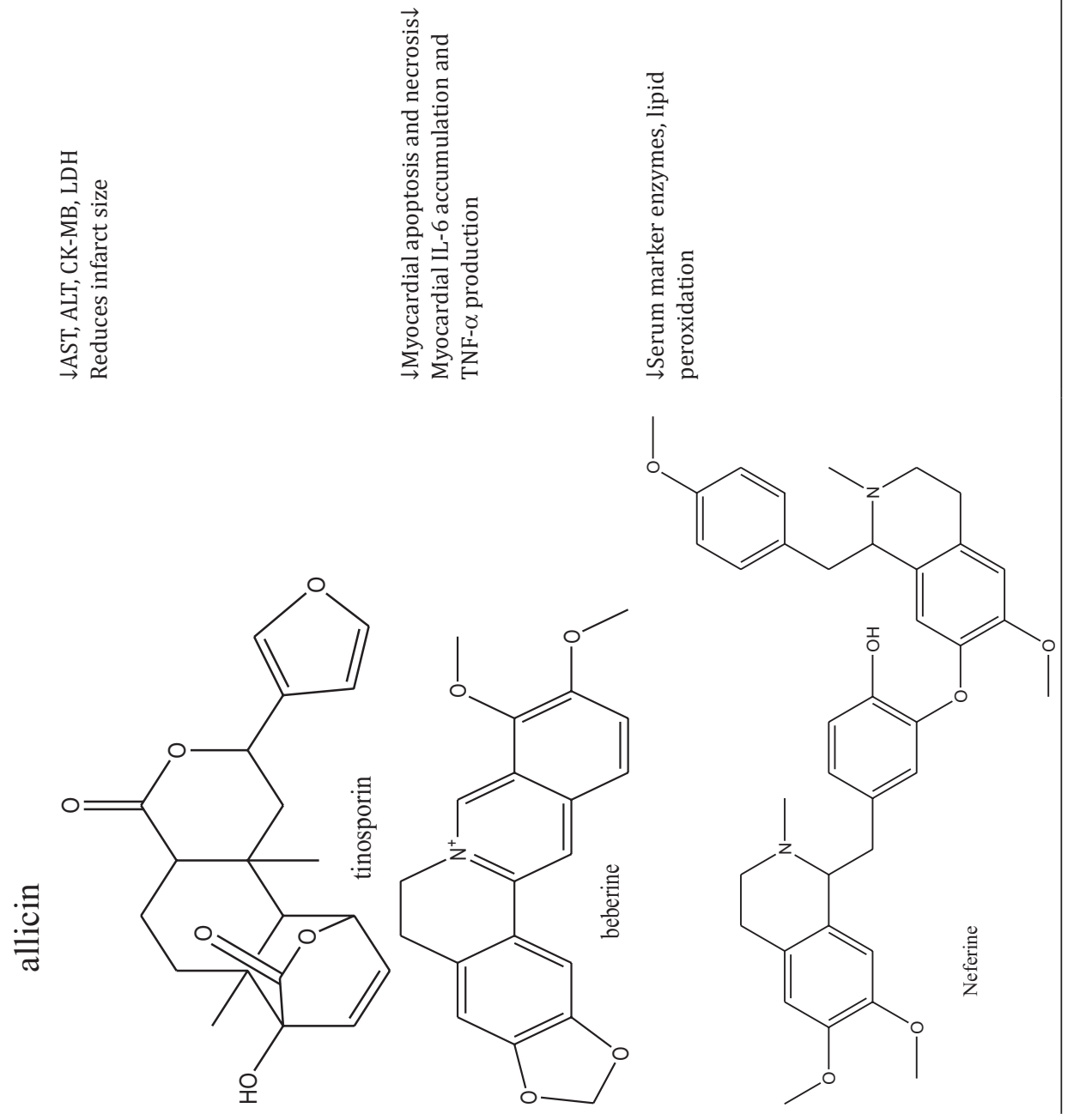

4

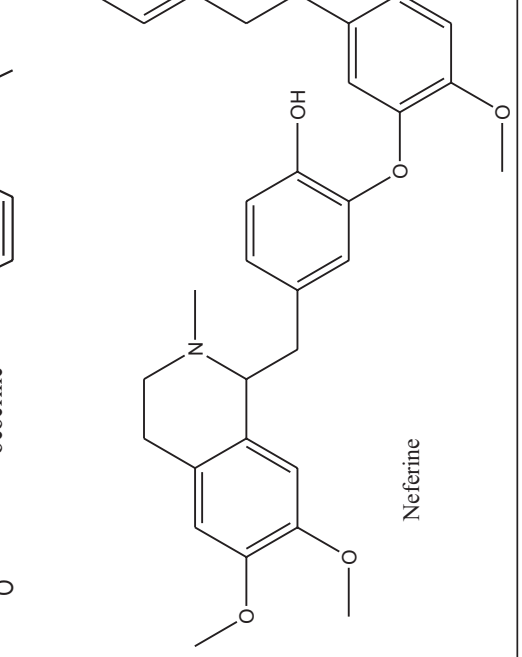

0
$\frac{0}{0}$
$\frac{0}{4}$
$\frac{\pi}{4}$

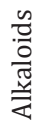

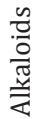
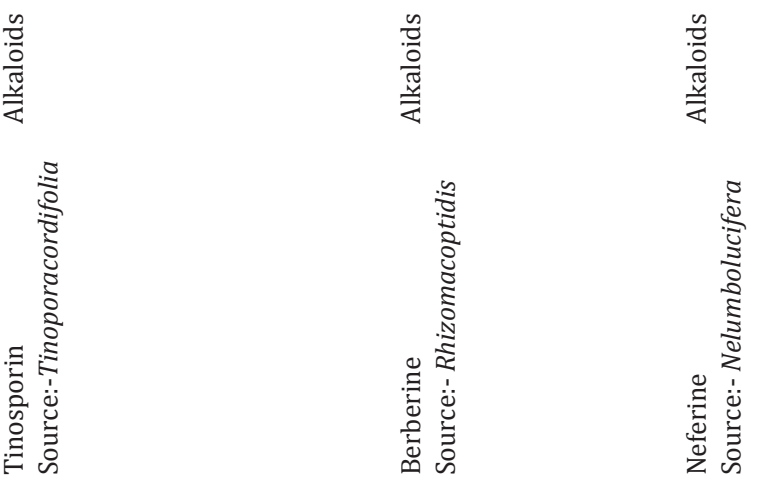


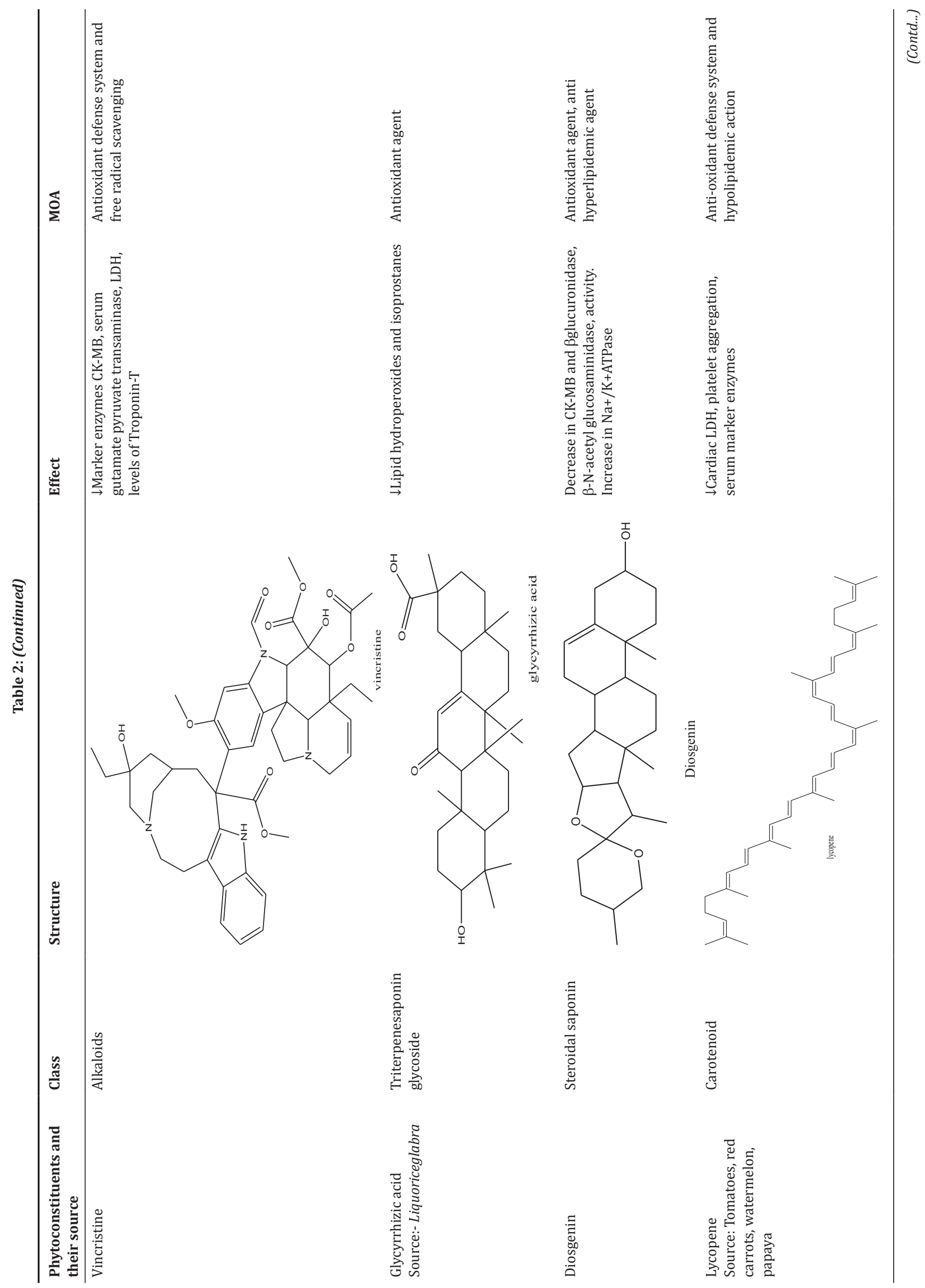




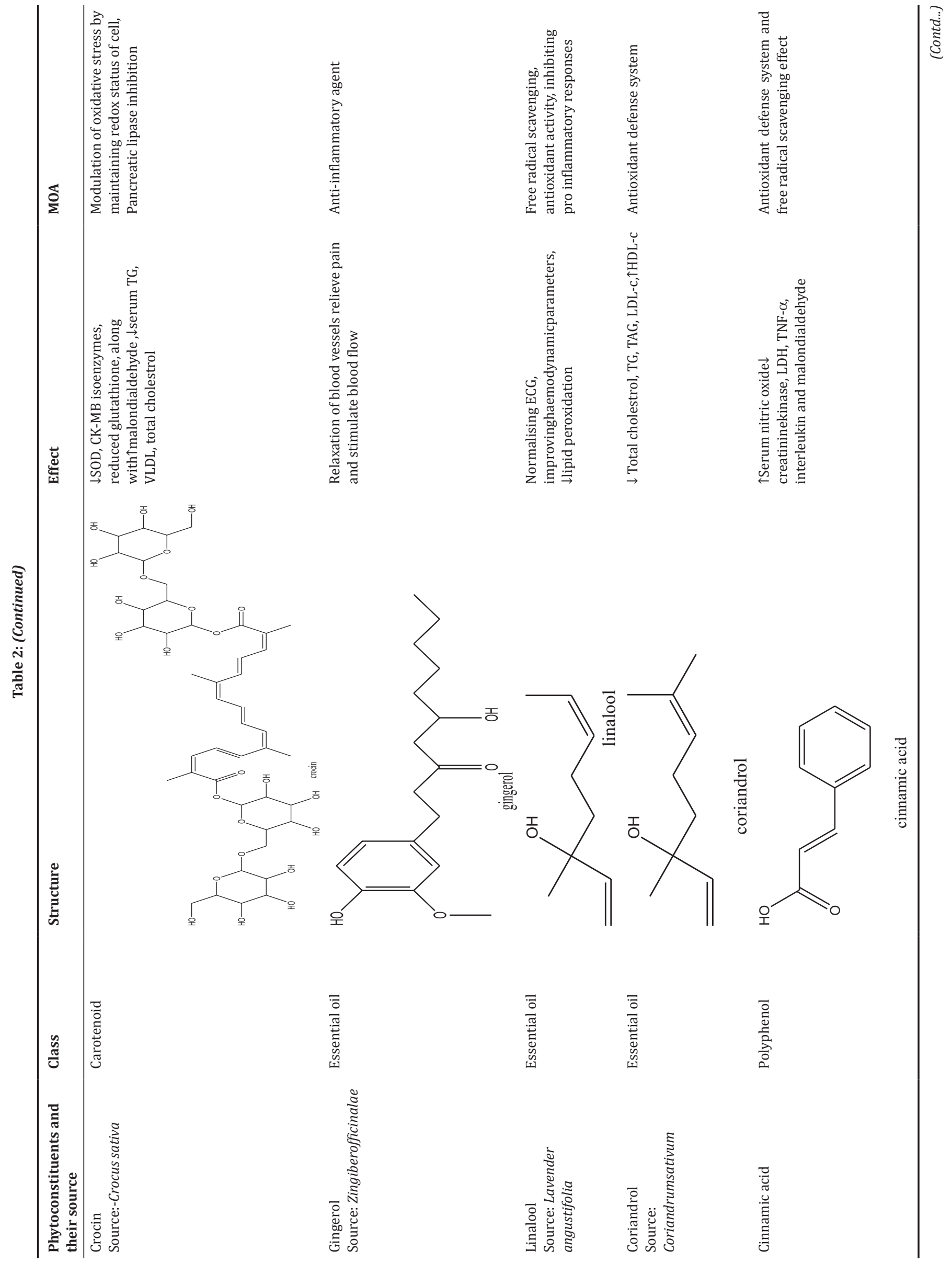



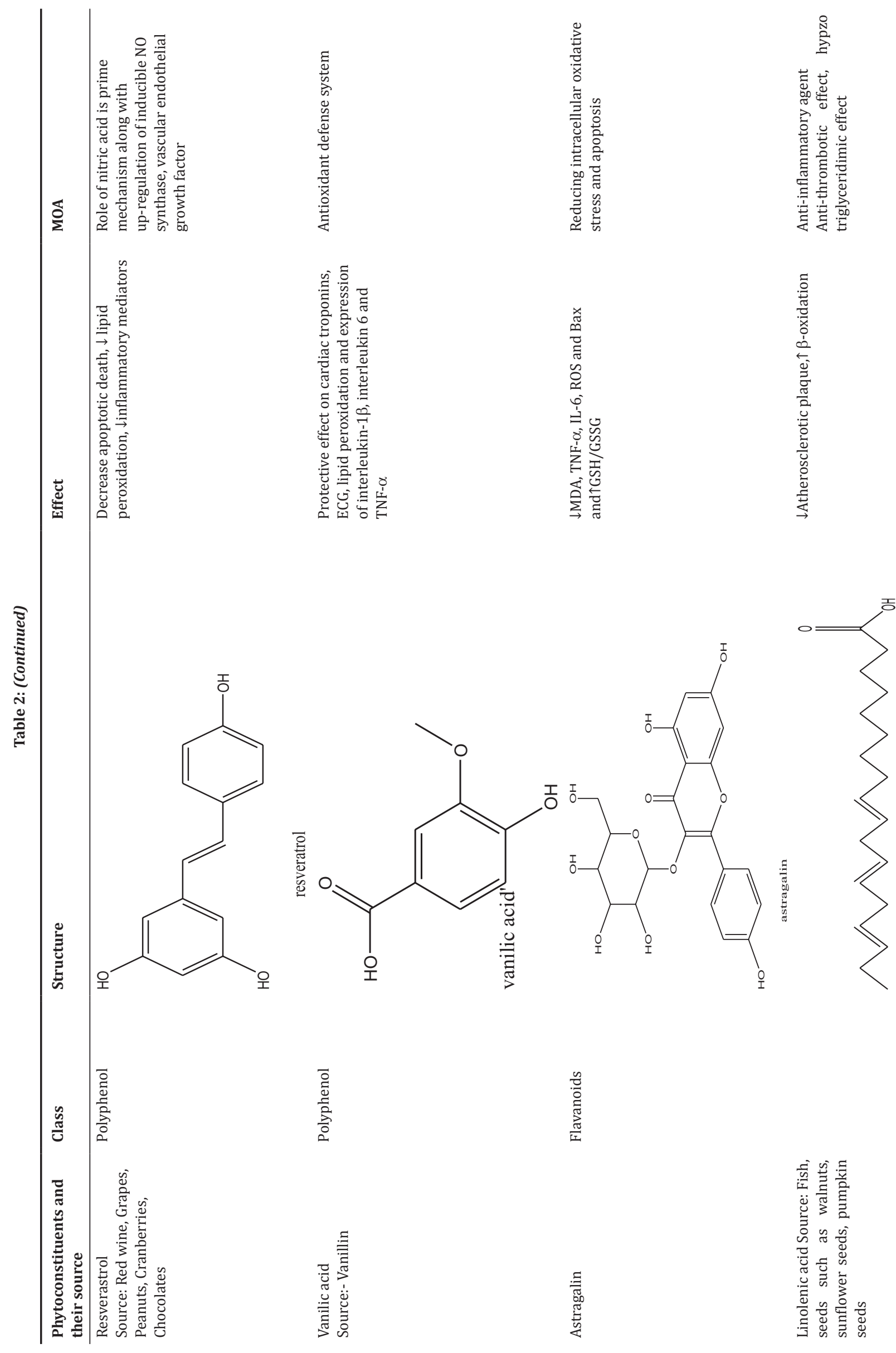

$\overrightarrow{0}$
$\frac{0}{0}$
$\frac{0}{2}$
$\frac{0}{0}$
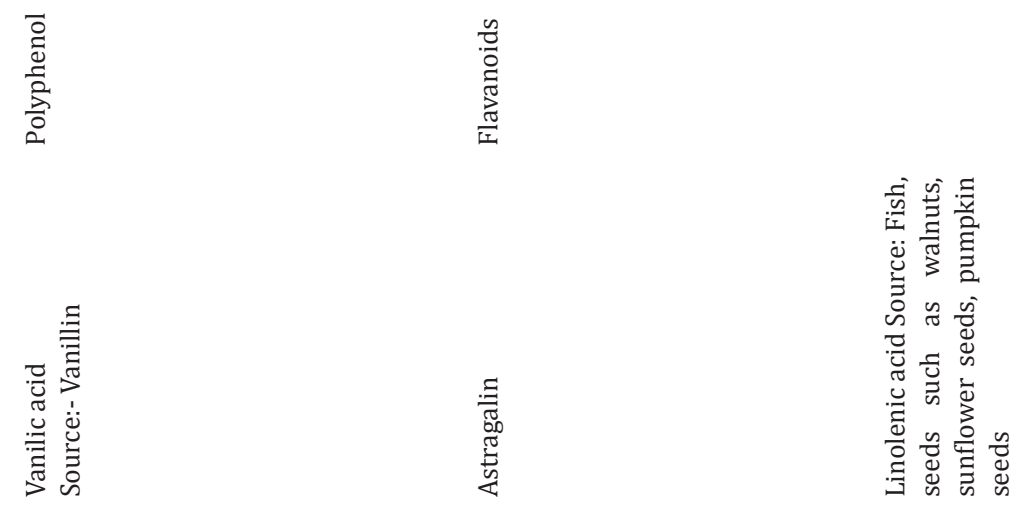
oil, corn oil, walnut, sunflower seeds, cashew nuts, etc. The sources from which omega 9 fatty acids are obtained include olive oil, almond oil, peanut oil, walnut, cashew, etc. [63]. Omega 3 fatty acids (Table 2) belong to a class of polyunsaturated fatty acids. The omega 3 fatty acids are concerned fundamentally with $\mathrm{CV}$ health are marine obtained eicosapentaenoic acid (EPA) and docosahexaenoic acid (DHA). The primary source includes fish oil and seafood which are obtained from the marine microorganisms which transmit through food chain. The plantprocured omega 3 fatty acids include linolenic acids which are further converted to EPA and DHA, but their role on cardioprotection is less clear than that of EPA and DHA [64]. It was presumed that the capability of these dietary fatty acids to exert pleotropic effects in cells and tissues is because they are embedded in the phospholipid of cellular membranes [65], resulting in the subsequent alteration of the physicochemical properties such as deformability, permeability [66], and fluidity. The antiinflammatory and anti-thrombotic effects of omega 3 fatty acids have been related to the metabolic conversion of their oxygenated derivatives which are predominantly named as oxylipins which are highly bioactive factors acting at very low concentrations [67]. The increased formation of specific LC-omega-3-PUFA is thought to decrease the atherosclerotic plaque formation and induce their stabilization by decreasing the infiltration of inflammatory and immune cells. The capability of $\omega-3$ PUFA to hinder the production of very LDLs (VLDLs) from the liver, as well as the transformation of VLDL to intermediate-density lipoprotein and LDLs, has been related to their hypotriglyceridemic effect. On the other hand, this effect has also been explained based on the potency of $\omega-3$ fatty acids to increase $\beta$-oxidation, thus leading to the depletion of the fatty acid substrate for triglyceride synthesis [68].

\section{Carotenoids}

Carotenoids, also known by the name tetraterpenoids, belong to a class of organic pigments commonly synthesized from plants, algae, bacteria, fungi, and are also present in foods such as fruits, vegetables, and fish. They are liposoluble pigments which are colorful in nature. Carotenoids include $\beta$-carotene, $\alpha$-carotene, lycopene, lutein, and cryptoxanthin. Carotenoids are responsible for the yellow, orange, and green color of fruits, flowers, and vegetables. Epidemiological studies conducted revealed that diet rich in carotenoids lowers the incidence of cancer, osteoporosis, CVDs and age-related macular degeneration, cataract, and infectious diseases such as HIV. The cardioprotective potential of carotenoids by altering various parameters is summarized in Fig. 2 [69].

Crocin

Crocus sativus L, a stemless herb commonly known by the name Saffron, is a member of the family Iridaceae. The pharmacologically active components of $C$. sativus include Saffranal, crocin, crocetin, and picrocrocetin. The major constituent of the essential oil of saffron includes Saffranal which is a monoterpene aldehyde and this, in particular, is responsible for the characteristic odor and aroma. The effects of crocin (Table 2), a pharmacologically active constituent of C. sativus L., in isoproterenol (ISO)-induced cardiotoxicity, were determined with reference to hemodynamic, antioxidant, histopathological, and ultrastructural parameters [55]. In vivo studies conducted with varying doses of aqueous saffron extract revealed a marked reduction in the levels of CK-MB isoenzyme, LDH, SOD, and reduced GSH along with an increase in the level of malondialdehyde (MDA). Under light microscopy, myocardial necrosis, edema, and inflammation were evident. Further, the protective role of crocin on ISO-induced myocardial damage was reconfirmed by histopathological and ultrastructural studies. Thus, the protective role of crocin in myocardial damage was more pronounced, and the mechanism in which it shows the action was by the modulation of oxidative stress by maintaining the redox status of the cell [70]. On administering aqueous extract of saffron, a remarkable reduction in the serum TGs, TC, and VLDL-cholesterol was observed. The hyperlipidemia effect of crocin was due to its pancreatic lipase inhibition [71].

Lycopene

Lycopene (Table 2), a red carotenoid pigment, is present abundantly in vegetables such as tomato, red carrot, watermelon, and papaya. Studies on lycopene revealed that it is the most efficient singlet oxygen quencher and peroxyl radical scavenger than other carotenoids and way better effective than $\alpha$-tocopherols. Studies have reported that lycopene protects adrenaline-induced MI, coronary artery disease, along with hypolipidemic and antioxidant activities. Studies have reported that simultaneous administration of Lycopene and Vitamin E has shown a decrease in the levels of cardiac marker enzymes, endogenous enzymes, membrane bound enzyme levels along with histopathological changes [72].

\section{Polyphenols}

Natural polyphenols are the broadest group of phytochemicals that gained much popularity as a potent candidate in the prevention of oxidative stress-related diseases such as diabetes, neurodegenerative disorders, cancer, CVDs, and aging [73,74]. They are secondary metabolites of plant, and the major source is plant-based foods. Polyphenols possess effect on bitterness, astringency, color, flavor, odor, and oxidative stability of food. Polyphenols act as potent antioxidant, anticancer, anti-aging, anti-inflammatory agent, and even show antimicrobial actions [30]. Polyphenols are generally divided into

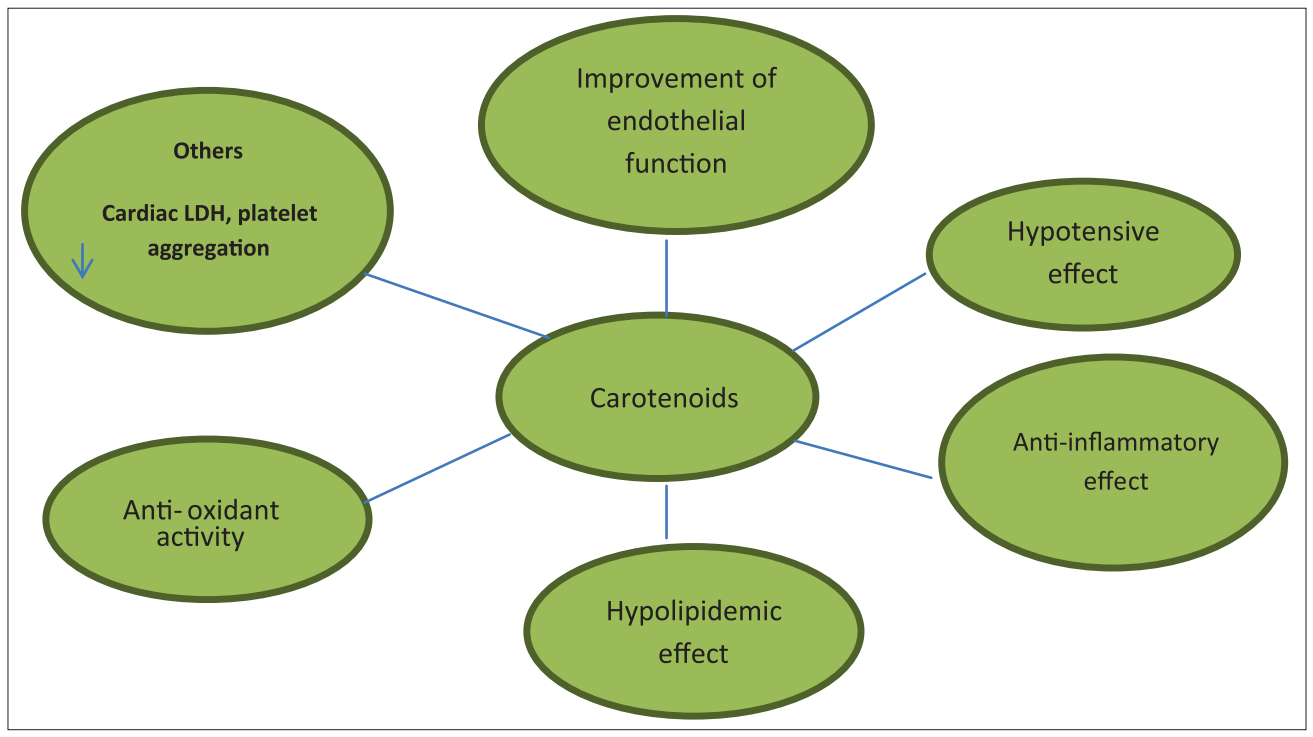

Fig. 2: Cardioprotective activity of carotenoids 
flavanoids and nonflavonoids: catechin, quercetin, proanthocyanidins, condensed tannins, and anthocyanins belong to flavanoids and hydrolyzable tannins, and benzene and cinnamate derivatives fall under nonflavanoids.

\section{Cinnamic acid}

Hydroxycinnamic acids (Table 2) are the prime class of polyphenols seen in every plant. The most indicative hydroxycinnamic acid is caffeic acid (3, 4-dihydroxy cinnamic acid) commonly seen in fruits, grains, and dietary supplements. It acts as a chelator of metal ion and shows free radical scavenging and antioxidant effects. In vivo studies conducted revealed that caffeic acid preserved the cardiac mitochondrial structure along with its functions in isoproterenol-induced MI. In vitro studies of caffeic acid revealed the free radical scavenging activity. The probable mechanism in which caffeic acid showed action is due to its antioxidant effect and free radical scavenging activity [75].

\section{Resveratrol}

Resveratrol (Table 2) belongs to the class of polyphenol which is beneficial to health in many ways because it has antioxidant and anticancer properties and also used in the treatment of CVDs. This is found in foods such as peanuts, pistachios, grapes, red wine, white wine, cranberries, blueberries, dark chocolate, and white chocolate [76]. In vivo studies demonstrated that pretreatment of RES resulted in protection against the deleterious effects of myocardial reperfusion after ischemia, especially by reducing the infarct size and decreasing arrhythmias. The role of nitric oxide (NO) is one of the prime mechanisms of pharmacological prerequisite by resveratrol. A current study proposed that the coordinated upregulation of inducible NO synthase, vascular endothelial growth factor, kinase insert domain-containing receptor, and endothelial NO synthase is one of the resveratrol's essential mechanisms. Resveratrol attenuates various soluble intercellular cytokines such as ICAM, VCAM, and E-selectin through upgrading the endothelium function, which lessens the infarct size [77]. Resveratrol also shields the heart by acting as a potent antioxidant by scavenging free radicals and inhibiting LPO both in vitro and in vivo [72]

\section{Vanilic acid}

Vanilic acid (Table 2), a polyphenolic acid, is the oxidized form of vanilin which is a widely used compound in drug, cosmetic, and food industries having various pharmacological activities such as antitumor [78], antimelangogenesis [79], and anti-angio genetic [80]. Vanilic acid acts as a natural antioxidant agent [81]. In vivo studies conducted revealed that pretreatment with vanilic acid showed significant protective effect on cardiac troponins, electrocardiogram, LPO, and expression of interleukin (IL) IL-1 $\beta$, IL-6, and tumor necrosis factor (TNF)- $\alpha$ gene in isoprenaline-induced cardiotoxicity in rats. The reduction in the size of infarct was also observed. The mechanism in which vanilic acid showed its effect is due to its possible antioxidant defense system [82].

\section{Flavanoids}

Flavanoids are structurally diverse and most abundant polyphenols. They are found in the form of glycosides and aglycosides. Flavanoids are divided into various subclasses which include flavanols, flavanones, flavones, isoflavones, and anthocyanidins. They are found in foods such as dark chocolates, red wine, citrus fruits, parsley, bananas, green tea, and onions with the content of $70 \%$ or more. The mechanisms of action of flavanols to show cardioprotective activity include antioxidant effect, modulation of cell signaling and gene expression, and modification of cell membrane properties and receptor function. In addition to the abovementioned properties, it can inhibit enzymatic activities [83].

\section{Astragalin}

Astragalin (Table 2), a bioflavonoid obtained naturally, has been well known for its pharmacological activity. It has been reported to exhibit multiple pharmacological properties including antioxidant, anti-inflammatory, anticancer, neuroprotective, and cardioprotective properties. The role in cardioprotective mechanism is due to the regulation of bradykinin, adenosine, opioid, adrenergic, and other G-protein-connected receptors. Astragalin was proved to be effective against acute ischemic reperfusion injury in Sprague-Dawley rats as its mechanism of action proceeds via reducing intracellular oxidative stress and apoptosis. The mechanism includes reduced expression of MDA, TNF- $\alpha$, IL- 6 , reactive oxygen species, and Bax along with the increased ratio of GSH/ oxidised glutathione (GSSG), respectively [84].

\section{Newer perspective}

CVDs, predominantly MI, is the leading cause of death globally. The increase in death rate is due to certain risk factors such as smoking, obesity, age, cholesterol, family history, diabetes, and sedentary lifestyle including lack of exercise and healthy food habits. The Statistics on Myocardial Infarction 2016 revealed that approximately 25,700 people suffered from an attack of acute MI. The study also revealed that the number of attacks and mortality rate in the public increases with age. Until the end of the $21^{\text {st }}$ century, mortality rate has been higher among men than among women regardless of education level. However, in recent years, mortality rate for men with postsecondary education is lesser than that for women with compulsory education. The treatment includes in MI is advanced and uses various methods and medication. The present-day treatment includes myocardial reperfusion where coronary thrombi were lysed by infusing streptokinase directly into the blocked coronary arteries of patients suffering from MI. In the past few years, myocardial reperfusion has advanced and, in addition to the use of aspirin, other antiplatelet agents to the fibrinolytics and stents - first being bare metals followed by drug-eluting stents - are on use. It is clearly observed that, along with the higher incidence of occurrences of diseases, the advancement in the treatment has been on point. However, as the saying goes "Prevention is better than cure," the incidence of occurring can be prevented with the help of nature. The above information revealed that nature is the best therapy because it contains various plants and plant-derived principles abundantly present in various forms. There are different ways in which the occurrence of disease can be prevented. It can be observed that, plantderived principles in different formulations can give a secondary protection to the myocardial tissues and can act as a supplement in aiding in the prevention of diseases. Usage of certain plants as a part of diet can improve the blood circulation, purify the blood, reduce the cholesterol level, and overall maintain a healthy body conditions. The field still remains open for the exploration of plant-based formulations that can not only offer protection against MI, but also serve as a therapeutic formulation to reverse the effects responsible for MI.

\section{CONCLUSION}

The findings of the study may help in elucidating the clinical mechanisms and effects of phytoconstituents underlying the valuable effects of plant-derived principles on CVDs, by the modulation of various cardiovascular risk factors such as oxidative stress, atherosclerosis, hyperlipidemia, and inflammation. Despite the previous studies in this area, this is a concise review which also considers the molecular mechanism of plant-derived principles in the cardiovascular system. Nevertheless, further systematic review and analysis are required to elucidate the exact mechanism of the beneficial effect.

\section{AUTHORS' CONTRIBUTION}

The first author contributed in the conceptualization of the article along with the collection of data and preparation of manuscript. The corresponding author provided expertise and feedback.

\section{CONFLICT OF INTERESTS}

We declare that we have no conflicts of interest.

\section{REFERENCES}

1. Kore KJ, Shete RV, Desai NV. Antiarthritic activity of hydroalcoholic extract of Lawsonia innermis. Int J Drug Dev Res 2011;3:217-24.

2. Shahana AA, Karale RS, Kamath JV. Cardioprotective effect of Mentha 
longifolia against cyclophosphamide induced cardiotoxicity in rats: A biochemical, electrocardiographic and histopathological study. Int J Pharm Pharm Sci 2016;8:214-7.

3. Kinattingal NK. Mruthunjaya A, Mahalakshmi M, Manjula SN. Cardioprotective effect of Tamarindus indica Linn against isoproterenol induced myocardial infarction in rats. Int J Pharm Pharm Sci 2016;8:254-60.

4. Hegstad AC, Ytrehus K, Myklebust R, Jørgensen L. Ultrastructural changes in the myocardial myocytic mitochondria: Crucial step in the development of oxygen radical-induced damage in isolated rat hearts? Basic Res Cardiol 1994;89:128-38.

5. Reddy KS. India wakes up to the threat of cardiovascular diseases. J Am Coll Cardiol 2007;50:1370-2.

6. Estruch R, Ros E, Salas-Salvadó J, Covas MI, Corella D, Arós F, et al. Primary prevention of cardiovascular disease with a Mediterranean diet. N Engl J Med 2013;368:1279-90.

7. Achan J, Talisuna AO, Erhart A, Yeka A, Tibenderana JK, Baliraine FN, et al. Quinine, an old anti-malarial drug in a modern world: Role in the treatment of malaria. Malar J 2011;10:144

8. Adeneyeab AA, Crooks PA. Weight losing, antihyperlipidemic and cardioprotective effects of the alkaloid fraction of Hunteria umbellata seed extract on normal and triton-induced hyperlipidemic rat. Asian Pac J Trop Biomed 2015;1691:30374-9.

9. Croaker A, King GJ, Pyne JH, Anoopkumar-Dukie S, Liu L. Sanguinaria canadensis: Traditional medicine, phytochemical composition, biological activities and current uses. Int J Mol Sci 2016;17:e1414.

10. Geleta B, Makonnen E, Debella A, Abebe A, Fekadu N. In vitro vasodilatory activity and possible mechanisms of the crude extracts and fractions of Moringa stenopetala (Baker f.) Cufod. Leaves in isolated thoracic aorta of guinea pigs. J Exp Pharmacol 2016;8:35-42.

11. Turabekova MA, Rasulev BF, Levkovich MG, Abdullaev ND, Leszczynski J. Aconitum and delphinium sp. Alkaloids as antagonist modulators of voltage-gated na+ channels. AM1/DFT electronic structure investigations and QSAR studies. Comput Biol Chem 2008;32:88-101.

12. Shoaib M, Shah SW, Ali N, Shah I, Ullah S, Ghias M, et al. Scientific investigation of crude alkaloids from medicinal plants for the management of pain. BMC Complement Altern Med 2016;16:178.

13. Kavitha D, Shilpa PN, Devaraj SN. Antibacterial and antidiarrhoeal effects of alkaloids of Holarrhena antidysenterica Wall. Indian J Exp Biol 2004;42:589-94.

14. Gushiken LF, Beserra FP, Rozza AL, Bérgamo PL, Bérgamo DA, Pellizzon $\mathrm{CH}$, et al. Chemical and biological aspects of extracts from medicinal plants with antidiabetic effects. Rev Diabet Stud 2016;13:96112.

15. Saha S, Ghosh S. Tinospora cordifolia: One plant, many roles. Anc Sci Life 2012;31:151-9.

16. Upadhyay AK, Kumar K, Kumar A, Mishra HS. Tinospora cordifolia (Willd.) hook. F. And Thoms. (Guduchi) validation of the ayurvedic pharmacology through experimental and clinical studies. Int J Ayurveda Res 2010;1:112-21.

17. Kesarwani N, Lubnaazmi A. Evaluation of cardioprotective effect of Tinospora cordifolia against isoprenaline induced myocardial infarction in rats. 2014;3:543-55.

18. Panda S, Kar A, Ramamurthy V. Cardioprotective effect of vincristine on isoproterenol-induced myocardial necrosis in rats. Eur J Pharmacol 2014;723:451-8.

19. Bhatt L, Sebastian B, Joshi V. Mangiferin protects rat myocardial tissue against cyclophosphamide induced cardiotoxicity. J Ayurveda Integr Med 2017;8:62-7.

20. Bradley JM, Organ CL, Lefer DJ. Garlic-derived organic polysulfides and myocardial protection. J Nutr 2016;146:403S-409S.

21. Pramod K, Ansari SH, Ali J. Eugenol: A natural compound with versatile pharmacological actions. Nat Prod Commun 2010;5:1999-2006.

22. Chennuru A, Saleem MT. Antioxidant, lipid lowering, and membrane stabilization effect of sesamol against doxorubicininduced cardiomyopathy in experimental rats. Biomed Res Int 2013;2013:934239.

23. Yesodharan K, Sujana KA. Ethnomedicinal knowledge among Malamalasar tribe of Parambikulam Wildlife Sanctuary, Kerala. Indian J Tradit Knowl 2007;6:481-5.

24. Srivastava KC, Bordia A, Verma SK. Curcumin, a major component of food spice turmeric (Curcuma longa) inhibits aggregation and alters eicosanoid metabolism in human blood platelets. Prostaglandins Leukot Essent Fatty Acids 1995;52:223-7.

25. Johnson WD, Morrissey RL, Usborne AL, Kapetanovic I, Crowell JA, Muzzio M, et al. Subchronic oral toxicity and cardiovascular safety pharmacology studies of resveratrol, a naturally occurring polyphenol with cancer preventive activity. Food Chem Toxicol 2011;49:3319-27.

26. Swaminathan JK, Khan M, Mohan IK, Selvendiran K, Devaraj SN, Rivera BK, et al. Cardioprotective properties of Crataegus oxycantha extract against ischemia-reperfusion injury. Phytomedicine 2010;17:744-52.

27. Zanwar AA, Hegde MV, Bodhankar SL. Cardioprotective activity of flax lignan concentrate extracted from seeds of Linum usitatissimum in isoprenalin induced myocardial necrosis in rats. Interdiscip Toxicol 2011;4:90-7.

28. Miceli C, Santin Y, Manzella N, Coppini R, Berti A, Stefani M, et al. Oleuropein aglycone protects against MAO-A-induced autophagy impairment and cardiomyocyte death through activation of TFEB. Oxid Med Cell Longev 2018;2018:8067592.

29. Kim JH, Yi YS, Kim MY, Cho JY. Role of ginsenosides, the main active components of Panax ginseng, in inflammatory responses and diseases. J Ginseng Res 2017;41:435-43.

30. Mattera R, Benvenuto M, Giganti MG, Tresoldi I, Pluchinotta FR, Bergante $\mathrm{S}$, et al. Effects of polyphenols on oxidative stress-mediated injury in cardiomyocytes. Nutrients 2017;9:e523.

31. de Souza CG, de Andrade DM, Jordão JB, de Ávila RI, Borges LL, Vaz BG, et al. Radical scavenger capacity of jabuticaba fruit (Myrciaria cauliflora) and its biological effects in hypertensive rats. Oxid Med Cell Longev 2017;2017:2383157

32. Shafiq H, Ahmad A, Masud T, Kaleem M. Cardio-protective and anticancer therapeutic potential of Nigella sativa. Iran J Basic Med Sci 2014; 17:967-79.

33. Jacques PF, Lyass A, Massaro JM, Vasan RS, D'Agostino RB Sr. Relationship of lycopene intake and consumption of tomato products to incident CVD. Br J Nutr 2013;110:545-51

34. Rao PR, Viswanath RK. Cardioprotective activity of silymarin in ischemia-reperfusion-induced myocardial infarction in albino rats. Exp Clin Cardiol 2007; 12:179-87

35. Kabera JN, Edmond S, Ally MR, Xin HE. Plant secondary metabolites: Biosynthesis, classification, function and pharmacological properties. J Pharm Pharmacol 2014;2:377-92.

36. Chatterjee K, Zhang J, Tao R, Honbo N, Karliner JS. Vincristine attenuates doxorubicin cardiotoxicity. Biochem Biophys Res Commun 2008:373:555-60.

37. Song F, Li H, Sun J, Wang S. Protective effects of cinnamic acid and cinnamic aldehyde on isoproterenol-induced acute myocardial ischemia in rats. J Ethnopharmacol 2013;150:125-30.

38. Sostaric T, Boyce MC, Spickett EE. Analysis of the volatile components in vanilla extracts and flavorings by solid-phase microextraction and gas chromatography. J Agric Food Chem 2000;48:5802-7.

39. Zhao X, Zhang J, Tong N, Liao X, Wang E, Li Z, et al. Berberine attenuates doxorubicin-induced cardiotoxicity in mice. J Int Med Res 2011;39:1720-7.

40. Wang Y, Liu J, MaA, Chen Y. Cardioprotective effect of berberine against myocardial ischemia/reperfusion injury via attenuating mitochondrial dysfunction and apoptosis. Int J Clin Exp Med 2015;8:14513-9.

41. Lv X, Yu X, Wang Y, Wang F, Li H, Wang Y, et al. Berberine inhibits doxorubicin-triggered cardiomyocyte apoptosis via attenuating mitochondrial dysfunction and increasing bcl-2 expression. PLoS One 2012;7:e47351.

42. Moses T, Papadopoulou KK, Osbourn A. Metabolic and functional diversity of saponins, biosynthetic intermediates and semi-synthetic derivatives. Crit Rev Biochem Mol Biol 2014;49:439-62.

43. Jurisić R, Debeljak Z, Vladimir-Knezević S, Vuković J. Determination of aucubin and catalpol in Plantago species by micellar electrokinetic chromatography. Z Naturforsch C 2004;59:27-31.

44. Singh D, Chaudhuri PK. Structural characteristics, bioavailability and cardioprotective potential of saponins. Integr Med Res 2018;7:33-43.

45. Karakida F, Ikeya Y, Tsunakawa M, Yamaguchi T, Ikarashi Y, Takeda S, et al. Cerebral protective and cognition-improving effects of sinapic acid in rodents. Biol Pharm Bull 2007;30:514-9.

46. Kar S, Palit S, Ball WB, Das PK. Carnosic acid modulates akt/IKK/ $N F-\kappa B$ signaling by PP2A and induces intrinsic and extrinsic pathway mediated apoptosis in human prostate carcinoma PC-3 cells. Apoptosis 2012;17:735-47

47. Haleagrahara N, Varkkey J, Chakravarthi S. Cardioprotective effects of glycyrrhizic acid against isoproterenol-induced myocardial ischemia in rats. Int J Mol Sci 2011;12:7100-13.

48. Lawson LD, Hunsaker SM. Allicin bioavailability and bioequivalence from garlic supplements and garlic foods. Nutrients 2018;10:e812.

49. Louis XL, Murphy R, Thandapilly SJ, Yu L, Netticadan T. Garlic extracts prevent oxidative stress, hypertrophy and apoptosis in cardiomyocytes: 
A role for nitric oxide and hydrogen sulfide. BMC Complement Altern Med 2012;12:140.

50. Jaradat NA, Zaid AN, Abuzant A, Shawahna R. Investigation the efficiency of various methods of volatile oil extraction from Trichodesma africanum and their impact on the antioxidant and antimicrobial activities. J Intercult Ethnopharmacol 2016;5:250-6.

51. Ziaee M, Khorrami A, Ebrahimi M, Nourafcan H, Amiraslanzadeh M, Rameshrad M, et al. Cardioprotective effects of essential oil of Lavandula angustifolia on isoproterenol-induced acute myocardial infarction in rat. Iran J Pharm Res 2015;14:279-89.

52. Hui L, He L, Huan L, Lan LX, AiGuo Z. Chemical composition of lavender essential oil and its antioxidant activity and inhibition against rhinitis related bacteria. Afr J Microbiol Res 2010;4:309-13.

53. Verma RS, Rahman LU, Chanotiya CS, Verma RK, Chauhan A, Yadav A. Essential oil composition of Lavandula angustifolia Mill. Cultivated in the mid hills of Uttarakhand, India. J Serb Chem Soc 2010;75:343-8.

54. Hassiotis CN, Lazari DM, Vlachonasios KE. The effects of habitat type and diurnal harvest on essential oil yield and composition of Lavandula angustifolia Mill. Fresenius Environ Bul 2010;19:1491-8.

55. Wakade AS, Shah AS, Kulkarni MP, Juvekar AR. Protective effect of Piper longum L. On oxidative stress induced injury and cellular abnormality in Adriamycin induced cardiotoxicity in rats. Indian J Exp Biol 2008;46:528-33.

56. Ramadan FM, Amer MM, Awad A, El-Sayed S. Coriander (Coriandrum sativum L.) seed oil improves plasma lipid profile in rats fed a diet containing cholesterol. Eur Food Res Technol 2008;21:319-28.

57. Pirali-Kheirabadi K, Teixeira da Silva JA. Lavandula angustifolia essential oil as a novel and promising natural candidate for tick (Rhipicephalus (Boophilus) annulatus) control. Exp Parasitol 2010;126:184-6.

58. Suneetha JW, Krishnakantha TP. Antiplatelet activity of coriander and curry leaf spices. Pharm Biol 2005;43:230-3.

59. Afzal M, Al-Hadidi D, Menon M, Pesek J, Dhami MS. Ginger: An ethnomedical, chemical and pharmacological review. Drug Metabol Drug Interact 2001;18:159-90.

60. Peter S, Chopra S, Jacob JJ. A fish a day, keeps the cardiologist away! A review of the effect of omega-3 fatty acids in the cardiovascular system. Indian J Endocrinol Metab 2013;17:422-9.

61. Bäck M. Omega-3 fatty acids in atherosclerosis and coronary artery disease. Future Sci OA 2017;3:FSO236.

62. Mehdizadeh R, Parizadeh MR, Khooei AR, Mehri S, Hosseinzadeh H. Cardioprotective effect of saffron extract and safranal in isoproterenolinduced myocardial infarction in Wistar rats. Iran J Basic Med Sci 2013;16:56-63.

63. Biondo PD, Brindley DN, Sawyer MB, Field CJ. The potential for treatment with dietary long-chain polyunsaturated n-3 fatty acids during chemotherapy. J Nutr Biochem 2008;19:787-96.

64. Stillwell W, Jenski LJ, Crump FT, Ehringer W. Effect of docosahexaenoic acid on mouse mitochondrial membrane properties. Lipids 1997;32:497-506.

65. Sansbury BE, Spite M. Resolution of acute inflammation and the role of resolvins in immunity, thrombosis, and vascular biology. Circ Res 2016;119:113-30.

66. Wu B, Mottola G, Schaller M, Upchurch GR Jr., Conte MS. Resolution of vascular injury: Specialized lipid mediators and their evolving therapeutic implications. Mol Aspects Med 2017;58:72-82.

67. Adkins Y, Kelley DS. Mechanisms underlying the cardioprotective effects of omega-3 polyunsaturated fatty acids. J Nutr Biochem 2010;21:781-92.

68. Milani A, Basirnejad M, Shahbazi S, Bolhassani A. Carotenoids: Biochemistry, pharmacology and treatment. $\mathrm{Br} \mathrm{J}$ Pharmacol 2017;174:1290-324

69. Mashmoul M, Azlan A, Khaza'ai H, Yusof BN, Noor SM. Saffron: A Natural potent antioxidant as a promising anti-obesity drug. Antioxidants (Basel) 2013;2:293-308.

70. Aman U, Vaibhav P, Balaraman R. Tomato lycopene attenuates myocardial infarction induced by isoproterenol: Electrocardiographic, biochemical and anti-apoptotic study. Asian Pac J Trop Biomed 2012;2:345-51.

71. Li AN, Li S, Zhang YJ, Xu XR, Chen YM, Li HB, et al. Resources and biological activities of natural polyphenols. Nutrients 2014;6:6020-47.

72. Bagchi D, Sen CK, Ray SD, Das DK, Bagchi M, Preuss HG, et al. Molecular mechanisms of cardioprotection by a novel grape seed proanthocyanidin extract. Mutat Res 2003;523-524:87-97.

73. Burns J, Yokota T, Ashihara H, Lean ME, Crozier A. Plant foods and herbal sources of resveratrol. J Agric Food Chem 2002;50:3337-40.

74. Vauzour D, Rodriguez-Mateos A, Corona G, Oruna-Concha MJ, Spencer JP. Polyphenols and human health: Prevention of disease and mechanisms of action. Nutrients 2010;2:1106-31.

75. Lirdprapamongkol K, Sakurai H, Kawasaki N, Choo MK, Saitoh Y, Aozuka Y, et al. Vanillin suppresses in vitro invasion and in vivo metastasis of mouse breast cancer cells. Eur J Pharm Sci 2005;25:57-65.

76. Chou TH, Ding HY, Hung WJ, Liang CH. Antioxidative characteristics and inhibition of alpha-melanocyte-stimulating hormone-stimulated melanogenesis of vanillin and vanillic acid from Origanum vulgare. Exp Dermatol 2010;19:742-50.

77. Sato M, Maulik G, Ray PS, Bagchi D, Das DK. Cardioprotective effects of grape seed proanthocyanidin against ischemic reperfusion injury. J Mol Cell Cardiol 1999;31:1289-97.

78. Lirdprapamongkol K, Kramb JP, Suthiphongchai T, Surarit R, Srisomsap C, Dannhardt G, et al. Vanillin suppresses metastatic potential of human cancer cells through PI3K inhibition and decreases angiogenesis in vivo. J Agric Food Chem 2009;57:3055-63.

79. Hegab MM, Ghareib HR. Antioxidative effects of acetone fraction and vanillic acid from Chenopodium murale $\mathrm{L}$ on tomato plant. Weed Biol Manag 2010;10:64-72.

80. Radmanesh E, Dianat M, Badavi M, Goudarzi G, Mard SA. The cardioprotective effect of vanillic acid on hemodynamic parameters, malondialdehyde, and infarct size in ischemia-reperfusion isolated rat heart exposed to PM $\mathrm{P}_{10}$. Iran J Basic Med Sci 2017;20:760-8.

81. Galleano M, Oteiza PI, Fraga CG. Cocoa, chocolate, and cardiovascular disease. J Cardiovasc Pharmacol 2009;54:483-90.

82. Keen CL, Holt RR, Oteiza PI, Fraga CG, Schmitz HH. Cocoa antioxidants and cardiovascular health. Am J Clin Nutr 2005;81:298S-303S.

83. Tilak-Jain JA, Devasagayam TP. Cardioprotective and other beneficial effects of some Indian medicinal Plants. J Clin Biochem Nutr 2006;38:9-18.

84. Qu D, Han J, Ren H, Yang W, Zhang X, Zheng Q, et al. Cardioprotective effects of astragalin against myocardial ischemia/Reperfusion injury in isolated rat heart. Oxid Med Cell Longev 2016;2016:8194690. 